\title{
VERIFICATION OF THE SPM IMPACT IN DWDM SYSTEM USING AWG MULTIPLEXER / DEMULTIPLEXER
}

\author{
Tomáš IVANIGA, Ján TURÁN, L'uboš OVSENÍK \\ Department of Electronics and Multimedia Telecommunications, Faculty of Electrical Engineering and Informatics, \\ Technical University of Košice, Vysokoškolská 4, 04001 Košice, Slovak Republic, Tel.: +421 55602 4277, \\ E-mail: tomas.ivaniga@tuke.sk, jan.turan@tuke.sk, lubos.ovsenik@tuke.sk
}

\begin{abstract}
This article describes the non-linear phenomenon SPM (Self Phase Modulation) located in all-optical communication systems. WDM systems are commercially available and providers use it without simulation. Also some experimental setups are available and simulation is not necessary because it offers real tests. The most important WDM components are AWG (Arrayed Waveguide Grating) multiplexer and demultiplexer where the multiplexing and demultiplexing of the optical signals happens. 10 Gbit/s optical line of the DWDM (Dense Wavelength Division Multiplex) system in accordance with ITU-G.694.1 was created and the SPM phenomenon is observed at it.
\end{abstract}

Keywords: $A W G, D W D M$, OptSim, SPM

\section{INTRODUCTION}

A very important aspect of fiber optical communication networks is that a single fiber can simultaneously transmit multiple signals of different wavelengths, which represent individual channels. In general, the most commonly used wavelengths are 1300$1600 \mathrm{~nm}$, which have the property of the smallest attenuation.

The technology that is used to merge multiple signals into a single optical fiber based on the wavelength is called Wavelength Division Multiplex (WDM Wavelength Division Multiplex) [1], [2], [3] and [4]. Conceptually, the WDM is based on Frequency Division Multiplexing FDM (Frequency Division Multiplex) used in the radio, or satellite technology. As with the FDM, each channel in the WDM must be determined by the wavelength, respectively a frequency separated from each other carefully, to avoid inter-channel interference [5]. These frequency bands between the channels are called borderlines. The WDM concept dates back to the year 1970. However, the research and development of this technology began only in 1977. In 1978, the WDM system was first implemented in the laboratory. This system could multiplex two signals. Today's modern WDM systems allow to multiplex up to 160 signals and expand $10 \mathrm{Gbit} / \mathrm{s}$ optical system to the theoretical capacity of $1.6 \mathrm{Tbit} / \mathrm{s}$. The Fig. 1 illustrates the principle of the WDM system.

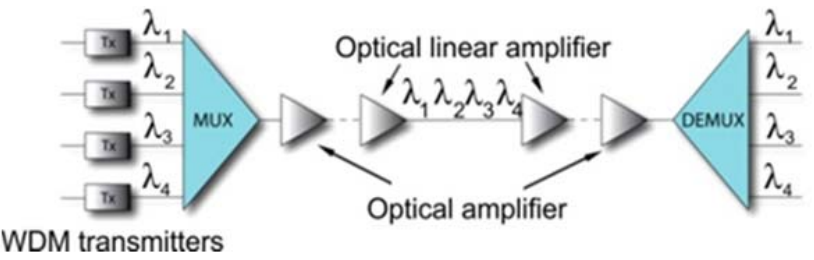

Fig. 1 The principle of the WDM system

The WDM system consists of a transmitting, optical and receiving part. The transmitting part is formed by the wave multiplexer, whose task is to combine optical signals from different sources to a single optical carrier. Another component is an optical amplifier which amplifies the optical signal for transmission over the optical fiber. The signal transmission is done directly in the optical fiber. On the receiving end is a demultiplexer that performs the inverse function of the multiplexer. Its mission is to spread the optical signal into the original sub-signals in optical form.

\section{OPTICAL KERR EFFECT AND SPM}

The basic equation defining the refractive index of the fiber core can be expressed by the following formula

$n=n_{0}+n_{2} \cdot \frac{P}{A_{e f}}$

where $n_{0}$ is the refractive index of the fiber core at a low power, $n_{2}$ is the nonlinear refractive index coefficient, $P$ is the optical power in Watts and $A e_{f}$ is the effective area of the fiber core given in square meters [5], [6]. On the basis of the following equation, it can be said that the refractive index of the optical fiber depends on the by level transmitted optical power which is proportional the transmitted light intensity of the fiber [5], [7]. The Fig. 2 shows that this variation is linear. For example refraction index 1.47003 is optical power equal to $60 \mathrm{~mW}$.

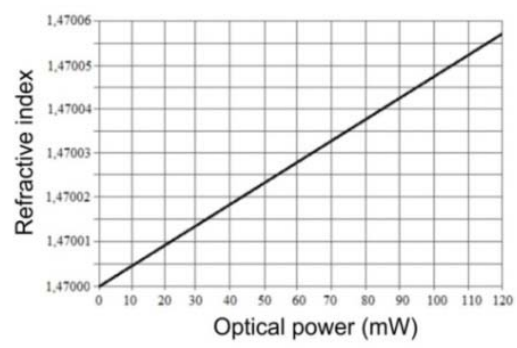

Fig. 2 The dependence of the refractive index of the optical fiber by level of the transmitted optical power

Depending on the shape of the input signal, the Kerr effect can be presented in different forms: SPM, FWM (Four-Wave Mixing) and XPM (Cross Phase Modulation). 
SPM refers to the phenomena when ultra-short light pulse guided by the transmission media results due to the Kerr effect into the change of the index of refraction of the transmission medium (optical fiber). The index of refraction causes a phase shift of the transmitted pulse. Therefore, the SPM is a very important phenomenon in optical communication systems using short pulses of light with high intensity radiation [8], [9] and [10]. The SPM phenomenon is particularly important in systems with high optical power.

For ultra-short light pulses with the Gaussian probability distribution and constant phase can be the intensity $I$ and time $t$ expressed by the following formula

$I(t)=I_{0} \cdot \exp \left(\frac{-t}{\tau}\right)$,

where $I_{0}$ is the value of maximum intensity and $\tau$ expresses half of the pulse duration.

When the pulse transits the medium, the optical Kerr effect causes a change in the refractive index that depends on the signal intensity $I$

$n(I)=n_{0}+n_{2}(I)$

In the formula (3), $n_{0}$ is the linear refractive index and $n_{2}$ is the nonlinear refractive index of the medium, which depends on the radiation intensity [11]. The refractive index of the core of the optical fiber is dependent on the intensity of the light emitted in the fiber.

When the light pulse passes the actual point transmission line, the impulse causes a certain intensity which afterwards drops to zero. This causes the refractive index of the medium, which is not constant along the transmission path. The index of refraction causes a shift in phase of the light pulse. The time-dependent phase of the pulse could be disclosed by the following formula

$\varphi(t)=\omega_{0}(t)-\frac{2 \pi}{\lambda_{0} n(I) L}$,

where $\omega_{0}$ is the frequency of the carrier, $\lambda_{0}$ is the wavelength of the pulse, $n$ is the refractive index and $L$ is the distance that the pulse has travelled. It can be therefore said that the layer is dependent on the intensity of the light pulse. This dependence leads to a change in the pulse spectrum [10], [11]. The Fig. 3 illustrates the change in frequency due to SPM.

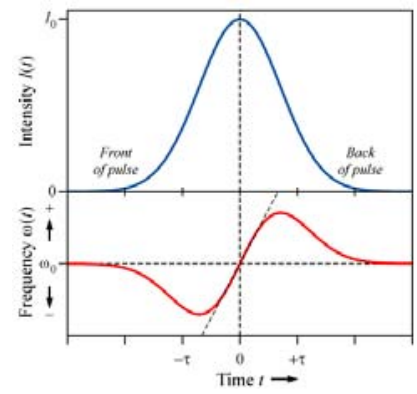

Fig. 3 The change in the frequency due to SPM [10]

From the graph it can be seen that in the middle of the pulse frequency is almost linear nature. The refractive index of the start-up pulse is increasing, leading to a decrease in the pulse rate. During pulse deceleration, the signal frequency increases due to a refractive index reducing [10]. These frequency changes are called frequency chirping. The frequency chirping extends the transmission of light pulses, thereby overlapping the pulse output. This phenomenon is known as dispersion. For systems with the transmission speed of $10 \mathrm{Gbit} / \mathrm{s}$ and more, but also for systems operating at a lower bit rate, the SPM can significantly increase the effect of chromatic dispersion.

When the SPM phenomenon and the effect of chromatic dispersion are approximately equivalent, but the chromatic dispersion in comparison with the SPM dominates, the SPM can reduce the effect of light pulses overlapping due to a chromatic dispersion [5], [7] and [11]. If the effects of chromatic dispersion and the SPM are equivalent, the pulses appear to be stable, so there is no overlap. Where the effect of chromatic dispersion is negligible, SPM causes the pulse amplitude modulation.

\section{ARRAYED WAVEGUIDE GRATINGS (AWG MUX/DEMUX)}

The key components of WDM systems are multiplexer and demultiplexer. The role of the multiplexer is to combine the wavelength of the optical signals from several sources (users) to one common transmission channel, which is an optical fiber [12]. The demultiplexer performs the inverse function of the multiplexer. Its role is to separate the signals of different wavelengths, which are intended for a particular recipient from the merger of the signal. For multiplexing in WDM systems, multiplexers / demultiplexers, which are referred as AWG (Arrayed Waveguide Gratings) could be used [12], [13]. These devices are characterized by low losses, high stability, relatively low cost and easy to implement in an integrated optical substrate.

\subsection{AWG principle}

The existing AWG multiplexers and demultiplexers are theoretically based on the diffraction and imaging principles. The AWG multiplexer is generally based on the MZI (Mach-Zehnder Interferometer) [13], [14]. The MZI is a device in which two copies of a single signal are phase-shifted by a certain stage and then merging together. Unlike the MZI, in the AWG are merged various phase copies of that signal. The AWG principally consists of four parts: array waveguides, input and output waveguides and FPR (Free Propagation Region) [13]. The Fig.4 shows the AWG configuration.

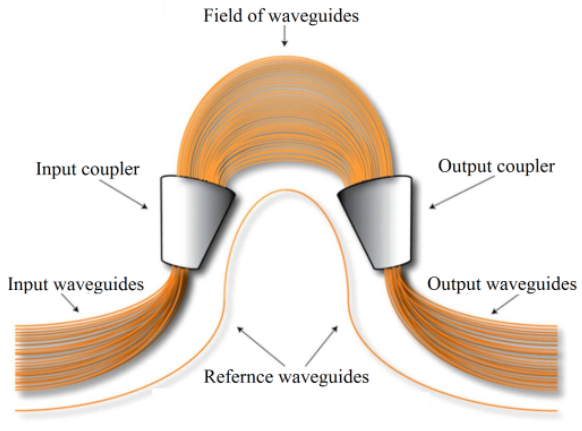

Fig. 4 The AWG configuration 
The number of input and output waveguides of AWG is $n$. The $m$ denotes the number of array waveguides which connect couplers at the input and output. Both couplers have then dimension $n m$. The difference in length between successive waveguides is constant and referred as $\Delta L$. The first coupler splits the input optical signal into $m$ parts.

The distance between the input $i$ and the array waveguides $k$ is $d^{i n}{ }_{i k}$. Similarly, $d^{\text {out }}{ }_{k j}$ represents the distance between the array waveguides and output $j$. Then the relative phase of the signal $\phi_{i j k}$ transmitted from input $i$ to output $j$ is defined by the following formula

$\phi_{i j k}=\frac{2 \pi}{\lambda}\left(n_{1} d_{i k}^{i n}+n_{2} k \Delta L+n_{1} d_{k j}^{o u t}\right)$

for $k=1,2, \ldots, m$.

In this relationship, $n_{l}$ is the refractive index areas of the input and output coupler, $n_{2}$ is the refractive index area of array waveguides and $\Delta L$ is the difference in length between successive specific waveguides [12], [15]. The input and output coupler can be designed as:

$d_{i k}^{i n}=d_{i}^{i n}+k \delta_{i}^{i n}$

$d_{k j}^{\text {out }}=d_{j}^{\text {out }}+k \delta_{j}^{\text {out }}$.

$\delta^{i n}{ }_{i}$ and $\delta^{\text {out }}{ }_{j}$ represent the distance from the input coupler to its output [16]. The relative phase $\phi_{i j k}$ of the signal transmitted from input $i$ and output $j$ can be modified to the following relationship

$\phi_{i j k}=\frac{2 \pi}{\lambda}\left(n_{1} d_{i}^{i n}+n_{1} d_{j}^{\text {out }}\right)+\frac{2 \pi k}{\lambda}\left(n_{1} \delta_{i}^{i n}+n_{2} \Delta L+\right.$ $\left.n_{1} \delta_{j}^{\text {out }}\right)$,

for $k=1,2, \ldots, m$.

Therefore, for the AWG multiplexer and demultiplexer the following applies:

$n_{1} \delta_{i}^{\text {in }}+n_{2} \Delta L+n_{1} \delta_{j}^{\text {out }}=p \lambda_{j}$

$n_{1} \delta_{i}^{\text {in }}+n_{2} \Delta L+n_{1} \delta_{j}^{\text {out }}=(p+1) \lambda_{j}^{\prime}$

wherein $p$ is an integer constant, and $\lambda_{j}$ and $\lambda_{j}^{\prime}$ are wavelengths demultiplexed at the output $j$.

\subsection{AWG multiplexer}

The AWG multiplexer configuration is shown in Fig. 5.

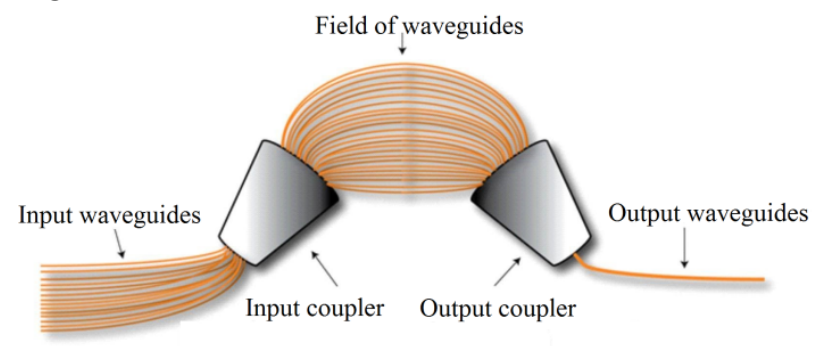

Fig. 5 The AWG multiplexer
The attenuation value for couplers is $-3 \mathrm{~dB}$. As already known from the previous theory, a copy of the input signals appears in the array of waveguides. The output signal $p\left(\lambda_{i}\right)$ is at the wavelength $\lambda_{i}$ for $i=1,2, \ldots, n$. It is also known that a number of array waveguide is $m$. Then the power $p\left(\lambda_{i}\right)$ can be divided into equal parts [12], [16]. Each part of the power is called $p^{\prime}\left(\lambda_{i}\right)$.

The output power of the multiplexer is

$P_{\text {in }}=\sum_{i=1}^{n} p\left(\lambda_{i}\right)$

where $n$ is the number of input signals, and $p\left(\lambda_{i}\right)$ is the power of the $i$-th signal.

The power at the output of the multiplexer can be expressed by the following relationship

$P_{\text {out }}=\sum_{i=1}^{n} a_{i} \cdot p^{\prime \prime}\left(\lambda_{i}\right)$,

where $a_{i}$ is the attenuation and $p$ ' $\left(\lambda_{i}\right)$ is the output for each wavelength $\lambda_{i}$ on the output port of the AWG multiplexer. This performance can be calculated by using following relationship

$p^{\prime \prime}\left(\lambda_{i}\right)=\sum_{k=1}^{l_{i}} b_{k} \cdot p^{\prime}\left(\lambda_{i k}\right)$

In this relationship is the number of waveguide lines $l_{i}$, attenuation $b_{k}$ and $p^{\prime}\left(\lambda_{i}\right)$ is the part of the power $p\left(\lambda_{i}\right)$. Hence the ratio of output and input power for a given wavelength $\lambda_{i}$ is expressed by the following formula

$\frac{P_{\text {out }}\left(\lambda_{i}\right)}{P_{\text {in }}\left(\lambda_{i}\right)}=\frac{\sum_{k=1}^{l_{i}} b_{k} \cdot p^{\prime}\left(\lambda_{i k}\right)}{p\left(\lambda_{i}\right)}$.

The total power at the multiplexer output is for each wavelength

$P_{M U X}=\frac{\sum_{i=1}^{n} a_{i} \cdot p^{\prime \prime}\left(\lambda_{i}\right)}{p\left(\lambda_{i}\right)}=\frac{\sum_{i=1}^{n} a_{i} \cdot\left[\sum_{k=1}^{l_{i}} b_{k} \cdot p^{\prime}\left(\lambda_{i k}\right)\right]}{p\left(\lambda_{i}\right)}$.

\subsection{AWG demultiplexer}

The AWG demultiplexer configuration is shown in Fig. 6 .

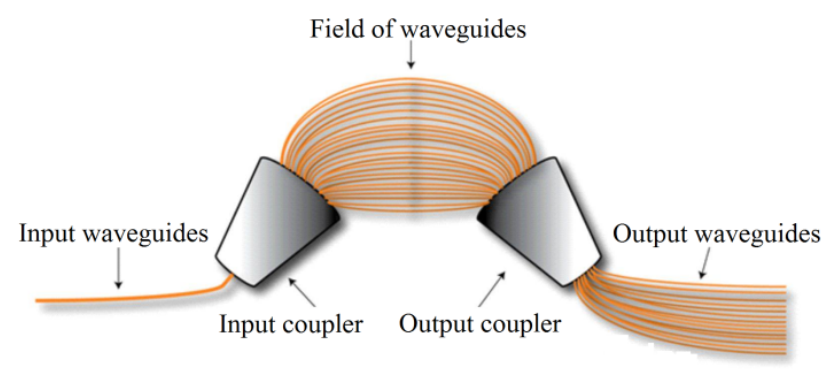

Fig. 6 The AWG demultiplexer

The input optical signal is in this case the sum of the performance of optical signals with different wavelengths [15], [16] and [19]. The total input optical power $P(\lambda)$ of the AWG demultiplexer is mathematically given by

$P(\lambda)=\sum_{i=1}^{n} p\left(\lambda_{i}\right)$ 
In this relationship is the optical power $p\left(\lambda_{i}\right)$ and the wavelength $\lambda_{i}$. The input coupler which size is $1 \times \mathrm{m}$, splits the input power $P(\lambda)$ to $m$ divisions. Each such part of the power $P(\lambda)$ is called $P^{\prime}(\lambda)$.

The optical power at the demultiplexer output $P\left(\lambda_{i}\right)_{\text {out }}$ is related to the wavelength $\lambda_{i}$ as follows

$P\left(\lambda_{i}\right)_{\text {out }}=\sum_{k=0}^{l_{i}} b_{k} p^{\prime}\left(\lambda_{i k}\right)=l_{i} \sum_{k=0}^{l_{i}} b_{k} p^{\prime}\left(\lambda_{i}\right)$.

The total output of the demultiplexer is the ratio of the output power $P\left(\lambda_{i}\right)_{\text {out }}$ and the total input power $P(\lambda)$

$P_{D E M U X}=\frac{P\left(\lambda_{i}\right)_{\text {out }}}{P(\lambda)}=\frac{l_{i} \sum_{k=0}^{l_{i}} b_{k} p^{\prime}\left(\lambda_{i}\right)}{\sum_{i=1}^{n} p\left(\lambda_{i}\right)}$.

\section{EXPERIMENTAL VERIFICATION OF THE SPM IMPACT IN AWG MUX/DMUX SYSTEMS}

The OptSim is a software environment that enables modelling and simulation of optical communication systems. It contains more than 400 algorithms representing a wide range of optical and optoelectronic components used in practice [16], [17] and [18]. Typical end-users in the OptSim environment are companies engaged in the development and implementation of network infrastructures to access a remote network [19].

\subsection{Draft model}

The Fig. 7 illustrates the two-channel DWDM system. This DWDM system contains 3 parts: transmitting part, optical part and receiving part.

\subsubsection{Transmitting part}

It consists of two DWDM channels. These channels include a data source called Datasource. It generates a pseudo-random sequence of bits at a rate of $10 \mathrm{Gbit} / \mathrm{s}$.
This sequence of bits is encoded in the block NRZ (NonReturn to Zero). The block generates the encoded NRZ electrical signal. The signal is modulated by MachZehnder modulator for optical carriers which source is a laser CW_Lorentzian $(6 \mathrm{dBm})$. The sources, i.e. laser diodes, emitting frequencies are 193.000 and $193.150 \mathrm{THz}$.

\subsubsection{Optical part}

The optical signal is amplified and transmitted by a single-mode optical fiber. The amplifier amplifies the input signal by $10 \mathrm{~dB}$. The OptSim indicates a non-linear effect SPM. At the output of the optical transmission part is the signal depredated due to SPM and dispersion and furthermore it is sent towards the recipient.

\subsubsection{Receiving part}

The output optical signal is converted to the electrical. The eye diagram, BER analysis, Q-factor, eye openness and jitter can be evaluated by the probes. The probe labelled as Input senses the signal before being transmitted through the optical communication system, with no SPM or dispersion impact.

In this simulation, it will be a reference probe, with which its results will be compared with the signal received at the output of the system. At the output of the probe is placed Scope. With this probe can be view the eye diagram and analyze BER, Q-factor, eye openness and jitter.

\subsection{Results of simulation}

In Fig. 8, 9 and 10 are shown the eye diagrams. In each case, there are two graphs, one without the SPM phenomenon and the other one with the SPM phenomenon. These charts show the change of the optical dispersion value affected by the SPM the transmission quality. The values of the dispersion are as follows: $-10,0$ and $10 \mathrm{ps} / \mathrm{nm} / \mathrm{km}$.

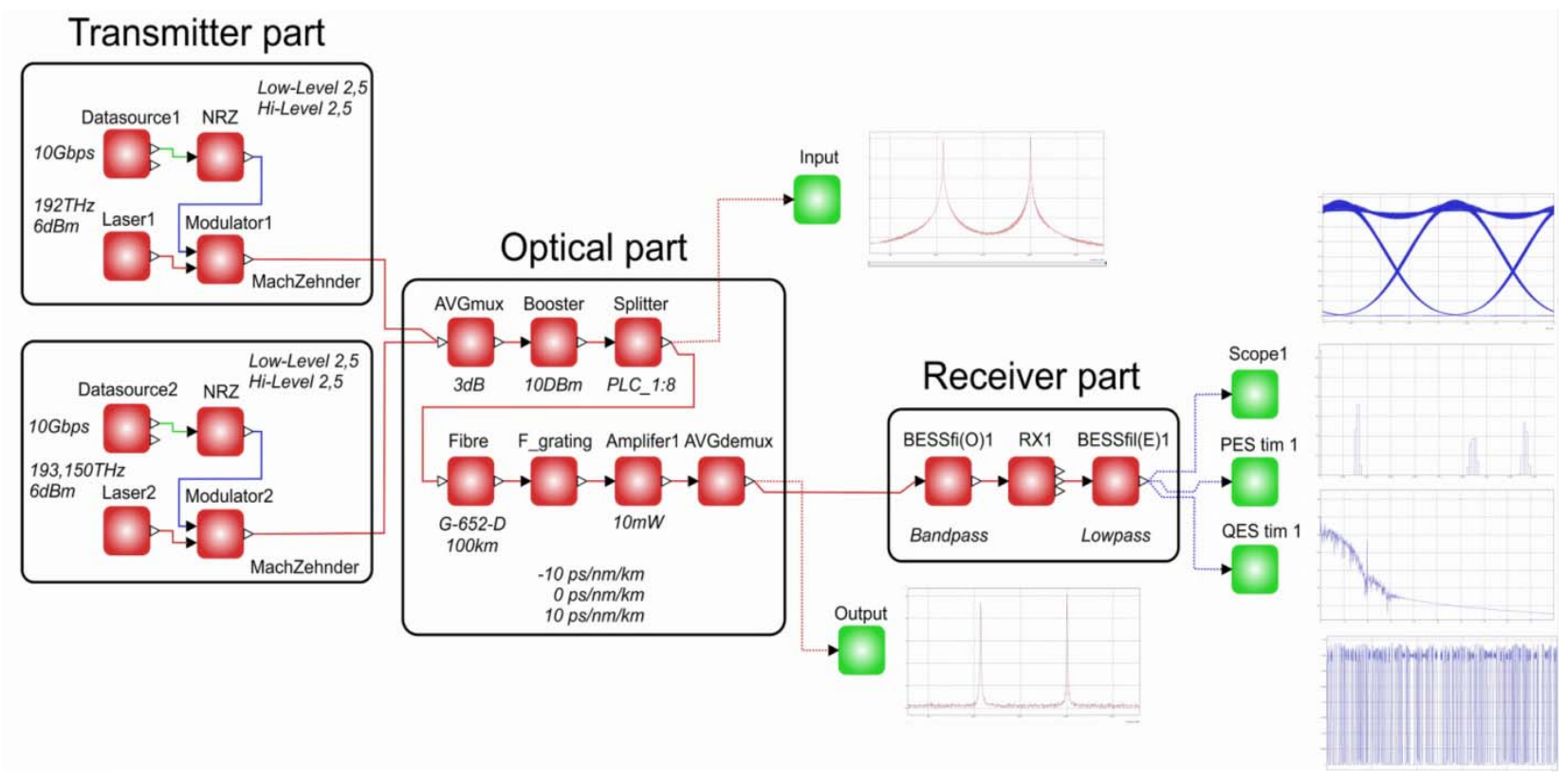

Fig. 7 The two-channel DWDM system 


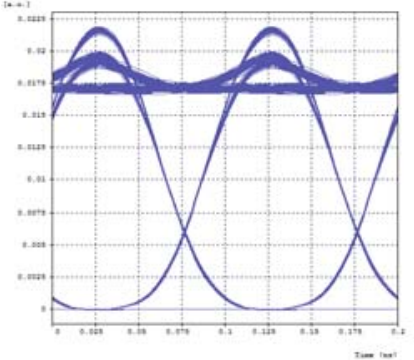

a)

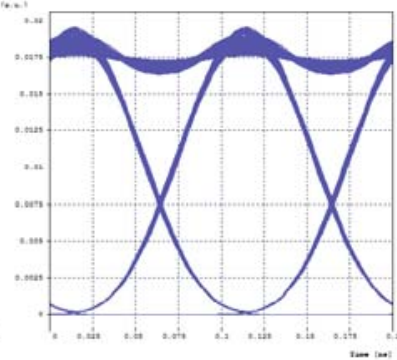

b)
Fig. 8 The eye diagram for the optical dispersion $D=-10$ (ps/nm/km) a) After the SPM (output) b) Before the SPM (input) a)

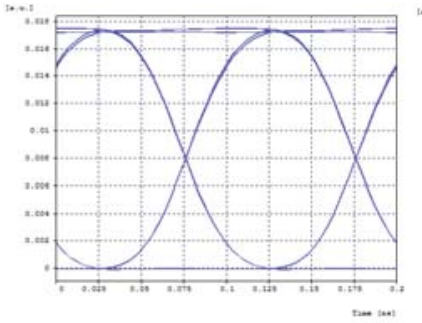

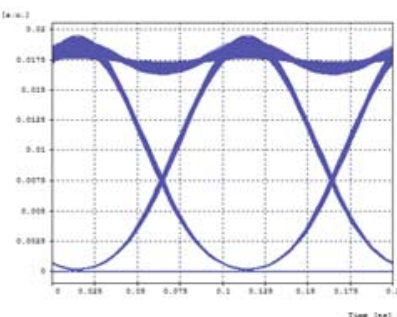

b)
Fig. 9 The eye diagram for the optical dispersion $\mathrm{D}=-0$ (ps/nm/km) a) After the SPM (output) b) Before the SPM (input)

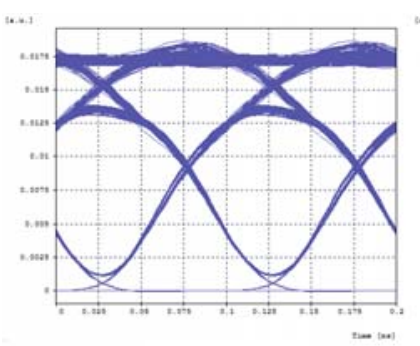

a)

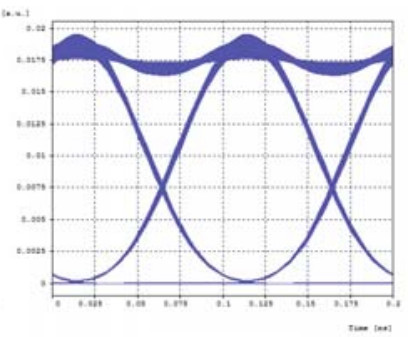

b)
Fig. 10 The eye diagram for the optical dispersion $D=10$ (ps/nm/km) a) After the SPM (output) b) Before the SPM (input)

The Fig. 11 refers to the Q-factor changes as a function of the dispersion.

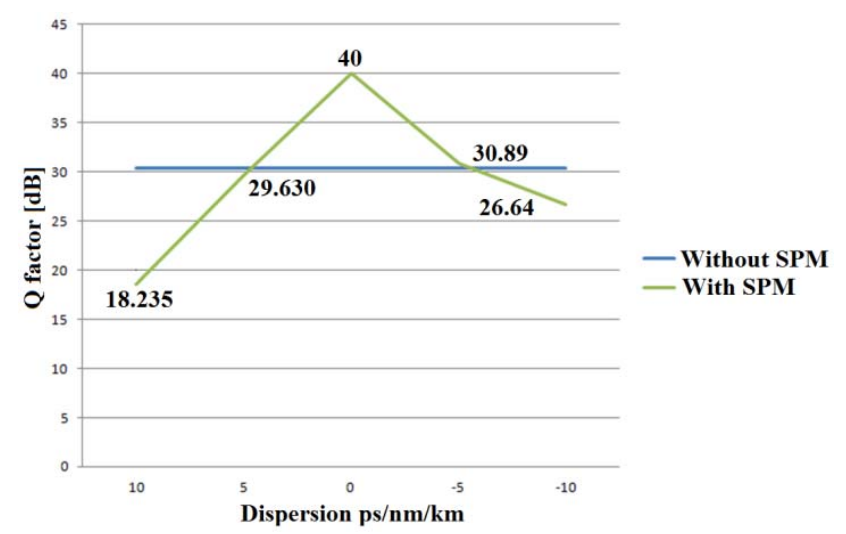

Fig. 11 The graph shows the Q-factor with the SPM and without the SPM

The resulting values are in Table 1 where the dispersion is changed with the increment 5 .
Table 1 Dispersion is changed with the increment 5

\begin{tabular}{|c|c|c|c|c|c|c|}
\hline \multicolumn{2}{|c|}{$\begin{array}{c}\text { Dispersion } \\
\text { (ps/nm/km) }\end{array}$} & 10 & 5 & 0 & -5 & -10 \\
\hline \multirow{2}{*}{$\begin{array}{c}Q \\
(\mathrm{~dB})\end{array}$} & $\begin{array}{l}\text { Without } \\
\text { SPM }\end{array}$ & \multicolumn{5}{|c|}{30,33816} \\
\hline & $\begin{array}{l}\text { With } \\
\text { SPM }\end{array}$ & 18,235 & 29,630 & 40 & 30,89 & 26,64 \\
\hline \multirow{2}{*}{ BER } & $\begin{array}{c}\text { Without } \\
\text { SPM }\end{array}$ & \multicolumn{5}{|c|}{$1 \mathrm{e}-040$} \\
\hline & $\begin{array}{l}\text { With } \\
\text { SPM }\end{array}$ & $1,8 \mathrm{e}-17$ & \multicolumn{4}{|c|}{$1 \mathrm{e}-040$} \\
\hline \multirow{2}{*}{$\begin{array}{c}\text { Jitter } \\
\text { (ns) }\end{array}$} & $\begin{array}{c}\text { Without } \\
\text { SPM }\end{array}$ & \multicolumn{5}{|c|}{0,00333483} \\
\hline & $\begin{array}{l}\text { With } \\
\text { SPM }\end{array}$ & 0,024 & 0,0243 & 0,02 & 0,015 & 0,017 \\
\hline
\end{tabular}

\section{CONCLUSION}

The aim of this article was to demonstrate the SPM non-linear effect which occurs in WDM systems. For implementing an optical communication system, it is necessary to take into account this phenomenon already in the proposal itself. In the simulation, the DWDM system was altered as it affects the SPM phenomenon dispersion. As it can be seen in Fig. 8, 9 and 10, the Q-factor became non-linear due to the SPM influence. The transmission quality characterized by the Q-factor was before the SPM phenomenon constant: 30.33816 . By changing the value of the dispersion, the Q-factor, jitter and BER have changed. The values of these parameters are shown in Table 1 .

\section{ACKNOWLEDGMENT}

This publication arose thanks to the support of the Operational Programme Research and development for the project "(Centre of Information and Communication Technologies for Knowledge Systems) (ITMS code 26220120020), co-financed by the European Regional Development Fund".

\section{REFERENCES}

[1] IVANIGA, P - MIKÚŠS, L.: Measuring of block error rates in high - speed digital networks, In: Advances in Electrical and Electronic Engineering, Vol. 5, No. 1, 2011, pp. 35-36, ISSN 1804-3119.

[2] MADAN, F. M. - KIKUCHI, K.: Design theory of long-distance WDM dispersion-managed transmission system, In: Journal of Lightwave Technology, Vol. 17, No. 8, 1999, pp. 1326-1335.

[3] IVANIGA, T. - OVSENÍK, L. - TURÁN, J.: Influence of Self-Phase Modulation on 8 and 16Channel DWDM System with NRZ and Miller Coding, In: Carpathian Journal of Electronic and Computer Engineering, Vol. 8, No.1, pp. 17-22, 2015, ISSN 1844-9689.

[4] ZISKIND, J. - SRIVASTAVA, A.: Optically Amplified WDM Networks, Academic Press-1 edition, 2011, 445 pp., ISBN 978-0-12-374965-9. 
[5] AGRAWAL, G. P.: Fiber-Optic Communication Systems, $4^{\text {th }}$ edition, John Wiley \& Sons, Inc., Hoboken, New Jersey, 2010, 626 pp., ISBN 978-0470-50511-3

[6] PIETRALUNGA, S. M. - MARTELli, P. FERRARIO, M. - MARTINELLI, M.: Measurement of self-phase modulation of amplified spontaneous emission: the role of second-order degree of coherence in determining the Kerr effect, in IEEE Photonics Technology Letters, Vol. 13, No. 11, Nov. 2001, pp. 1179-1181.

[7] ISLAM, S. M - DEWANJEE, A. - SULTANA, M.: Cross-Phase and Self-Phase Modulation: Effect on the Performance of a WDM Link, VDM Verlag Dr. Müller, Saarbrucken, Germany, 2010, 84 pp., ISBN13: 978-3-639-24841-8.

[8] LIN, G. - DIALLO, S. - CHEMBO, Y. K.: Optical Kerr frequency combs: Towards versatile spectral ranges and applications, 2015, 17th International Conference on Transparent Optical Networks (ICTON), Budapest, 2015, pp. 1-4.

[9] DUILL, S. O. - O'DOWD, R.: Impact of Kerr nonlinearity on novel orthogonal modulation formats in WDM networks, 2009 International Conference on Photonics in Switching, Pisa, 2009, pp. 1-2.

[10] IVANIGA, P.: Error Rate Characteristics for MultiOperator Transmission Path, In: Elektrorevue, Vol. 15, No. 1, 2013, pp. 1-4, ISSN 1213-1539.

[11] UDALCOVS, A. - BOBROVS, V. - PORINS, J.: Evaluation of SPM-induced optical signal distortions in ultra-dense mixed-WDM system, Future Generation Communication Technology (FGCT), 2012 International Conference on, Dec. 2012, pp. $12-14$.

[12] TÓTH, J. - OVSENÍK, L'. - TURÁN, J.: Free space optics experimental system - long term measurements and analysis, In: Acta Electrotechnica et Informatica, Vol. 15, No. 2, 2015, pp. 26-30.

[13] SEYRINGER, D. - UHEREK, F. - CHOVAN, J. KUZMA, A.: Design, simulation and evaluation of AWG based demultiplexers, Advanced Semiconductor Devices \& Microsystems (ASDAM), 2012 Ninth International Conference on, Smolenice, 2012, pp. 303-306.

[14] KAWAI, T. - OBARA, H.: Crosstalk reduction in $\mathrm{N} \times \mathrm{N}$ WDM multi/demultiplexers by cascading small arrayed waveguide gratings (AWG's), In: Journal of Lightwave Technology, Vol. 15, No. 10, Oct. 1997, pp. 1929-1937.

[15] OVSENÍK, L'. - KAŽÍMÍROVÁ KOLESÁROVÁ, A. - TURÁN, J.: Video Surveillance Systems, In: Acta Electrotechnica et Informatica, Vol. 10, No. 4, 2010, pp. 46-53.
[16] IVANIGA, P. - MIKÚŠ, L.: The Realtionship Between G.826 Error Performance Objectives and Equivalent BER, In: Journal of Information, Control and Management Systems, Vol. 2, No. 1, 2007, pp. 39-44, ISSN 1336-1716.

[17] Rsoft Design Group, Inc: Manual of OptSim Models Refernce Volume 1 Sample Mode, Physical Layer Division, 400 Executive Boulevard, Suite 100, Ossining, NY 10562, 2009, 543 pp.

[18] ABDUllaEV, A. - TURÁN, J.: Survey of the Problems and Solutions of Arrayed Waveguide Gratings Used in the Optical Networks, In: Acta Electrotechnica et Informatica, Vol. 14, No. 3, 2014, pp. $49-53$.

[19] SUJECKI, S.: Photonics Modelling and Design, CRC Press, Taylor \& Francis Group, 6000 Broken Sound Parkway NW, Suite 300, 2015, 393 pp., ISBN-13: 978-1-4665-6126-7.

Received September 3, 2016, accepted January 12, 2017

\section{BIOGRAPHIES}

Tomáš Ivaniga received Ing. (MSc) degree from "Infoelectronics" University of Košice in 2014 at Department of Electronics and Multimedia Telecommunications, Faculty of Electrical Engineering and Informatics. He has started his PhD. studies at Technical University of Košice on September 2014. His main field of study is a research of full optical fiber networks, mitigation and degradation mechanisms in fully optical networks.

Ján Turán (Dr.h.c., prof., RNDr., Ing., DrSc.) received Ing. (MSc.) degree in physical engineering with honours from the Czech Technical University, Prague, Czech Republic, in 1974, and RNDr. (MSc.) degree in experimental physics with honours from Charles University, Prague, Czech Republic, in 1980. He received a CSc. (PhD.) and DrSc. degrees in radioelectronics from University of Technology, Košice, Slovak Republic, in 1983, and 1992, respectively. Since March 1979, he has been at the University of Technology, Košice as Professor for electronics and information technology. His research interests include digital signal processing and fiber optics, communication and sensing.

Luboš Ovseník (doc., Ing., PhD.) received Ing. (MSc.) degree in radioelectronics from the University of Technology, Košice, in 1990. He received PhD. degree in electronics from University of Technology, Košice, Slovak Republic, in 2002. Since February 1997, he has been at the University of Technology, Košice as Associate Professor for electronics and information technology. His general research interests include optoelectronic, photonics, fiber optic communications and fiber optic sensors. 